\title{
Noninvasive radioelectric asymmetric brain stimulation in the treatment of stress-related pain and physical problems: psychometric evaluation in a randomized, single-blind placebo-controlled, naturalistic study
}

This article was published in the following Dove Press journal: International Journal of General Medicine

2I September 20II

Number of times this article has been viewed

\author{
Vania Fontani' \\ Salvatore Rinaldi ${ }^{1,2}$ \\ Lucia Aravagli' \\ Piero Mannu' \\ Alessandro Castagna' \\ Matteo Lotti Margotti' \\ 'Rinaldi-Fontani Institute, ${ }^{2}$ Medical \\ School of Occupational Medicine, \\ University of Florence, Florence, Italy
}

Background: The aim of this study was to investigate the effects of noninvasive radioelectric
asymmetric conveyer brain stimulation (REAC-BS) on pain and physical problems, a
measurement cluster of the Psychological Stress Measure (PSM) test. When the symptoms of
pain and physical problems do not respond to various therapeutic approaches such as medication,
physiotherapy, and psychotherapy, they are often called medically unexplained symptoms. As
such, these symptoms are reported to be a response to stressful situations or emotional states,
often unknown to patients themselves. To explore the effectiveness of noninvasive radioelectric
brain stimulation in the amelioration of symptoms of pain and physical problems, we adminis-
tered a neuropsychophysical optimization protocol using a REAC device. Methods: The PSM, a self-administered questionnaire, was used to measure psychological stress and pain and physical problems in a group of 888 subjects. Data were collected immediately prior to and following a 4-week REAC treatment cycle.

Results: There was a significant reduction in scores measuring subjective perceptions of stress for subjects treated with one cycle of neuropsychophysical optimization REAC-BS. At the end of the study, the number of treated subjects reporting symptoms of stress-related pain and physical problems on the PSM test was significantly reduced, whereas there was no difference in placebo-treated subjects.

Conclusion: One cycle of neuropsychophysical optimization REAC-BS appears to reduce subjective perceptions of stress as measured by the PSM, particularly on the pain and physical problems cluster.

Keywords: stress disorders, adaptation disorders, medically unexplained symptoms

\section{Introduction}

Pain and physical problems of various kinds which display a chronic course and do not meet diagnostic criteria for specific treatments ${ }^{1}$ are generally defined as medically unexplained symptoms. ${ }^{2-4}$ These symptoms may be present in people of all ages and social groups, ${ }^{5}$ and represent a difficult problem for both general practitioners ${ }^{6-8}$ and specialists. ${ }^{9}$ Pain and physical problems and medically unexplained symptoms (PPP-MUS) are often considered to be, and treated as, psychiatric disorders. ${ }^{6,10-12}$ However, it may be more accurate to consider PPP-MUS as stress-related maladaptive illnesses ${ }^{13-15}$ or psychosomatic disorders, which are classified as "psychological factors
Correspondence: Salvatore Rinald Rinaldi Fontani Institute, Viale Belfiore 43, 50144 Florence, Italy Tel +39055290307

Fax +390 55290399

Email srinaldi@irf.it 
affecting medical condition" in the DSM-IV (Diagnostic and Statistical Manual of Mental Disorders, Fourth Edition, Text Revision). ${ }^{16}$ The most common PPP-MUS include migraine, headaches, neck and low back pain, and joint pain. Usually the treatment plan for medically unexplained symptoms involves a combination of behavior modification and pharmaceutical treatment, as well as good communication between patient and doctor. The purpose of the current work is to determine whether the use of noninvasive radioelectric asymmetric conveyer brain stimulation (REAC-BS) is effective in reducing subjective perceptions of stress as measured by the Psychological Stress Measure (PSM) test, a validated questionnaire ${ }^{17-19}$ allowing for the classification of a subject's stress level and stress-related symptoms of pain and physical problems.

\section{Materials and methods}

\section{Participants}

Eight hundred and eighty-eight subjects were included in the study from an initial group of 1453 patients (Supplement 1) who attended the Rinaldi-Fontani Institute in Florence, Italy. These patients presented with different types of stress-related PPP-MUS such as migraine, headaches, neck and low back pain, joint pain, and functional somatic pain syndrome. In all subjects, the pain had been present for several years, and patients had found little or no benefit from previous treatments including drugs, physiotherapy, or any of several psychotherapeutic approaches. The subjects included in the study were taking pain medication as needed for unbearable pain, but were asked not to take specific psychotropic drugs. The study was performed in accordance with the Declaration of Helsinki.

\section{Sample size and randomization}

This was a naturalistic study, so patients with stress-related pain and physical problems came unsolicited to our private medical center and were observed in normal clinical practice. To obtain a sample of control subjects for comparison with the treated patients, two groups were created. Subjects were randomly assigned to each group using simple computerized randomization by an external operator. Group A subjects received active treatment and Group B subjects received placebo treatment, ie, inactive REAC, in a specific room, in an approximately $3: 1$ ratio.

\section{Demographic characteristics}

Group A included 688 subjects comprising 401 (58.3\%) females, of average age $42.3 \pm 11.3$ years, and $287(41.7 \%)$ males of average age $41.1 \pm 11.4$ years. These patients were treated with active REAC. Group B included 200 subjects comprising $123(61.5 \%)$ females of average age $48.8 \pm 19.4$ years, and $77(38.5 \%)$ males of average age $45.8 \pm 18.5$ years. These patients were treated with inactive REAC (see Table 1).

\section{Psychological and psychiatric assessment}

The PSM was developed specifically to detect stress levels in a nonclinical population. ${ }^{17-19}$ The PSM usually consists of a 49-item self-report pencil and paper questionnaire. In this study, we used an electronic version to collect and process the data, and to analyze the results. Patients were asked to answer questions about their psychological stress using a four-point scale to describe the intensity of their condition (very $\operatorname{much}=4, \operatorname{much}=3$, little $=2$, none $=1$ ). The final score is expressed in total points. To detect the presence of symptoms of pain and physical problems, scores were specifically obtained from questions referring to difficulty with digestion, stomach pain, feelings of a knot in the stomach (question 12), physical aches and pains, including a sore back, headache, stiff neck, stomach ache (question 14), diarrhea or intestinal cramping or constipation (question 28). Both groups of subjects at time 0 were examined by a psychiatrist to detect possible psychiatric disorders that could affect symptoms of pain and physical problems. The average total PSM test score was $122.53 \pm 6.75$ for Group A and $122.96 \pm 7.041$ for Group B.

\section{REAC technology and therapeutic protocol}

REAC is an innovative medical technology for bioenhancement. ${ }^{20,21}$ REAC-specific treatments have proven efficacious in ameliorating stress-related disorders, ${ }^{22-25}$ depression, ${ }^{26}$ anxiety, ${ }^{25,27}$ bipolar disorder, ${ }^{28}$ and other psychiatric disorders. ${ }^{29}$ REAC administration has also been shown to be effective in treating neuromotor dysfunction, ${ }^{30}$ posttraumatic pain and injury, ${ }^{31,32}$ and improving functional recovery in arthritic lower limb joints. ${ }^{33,34}$ REAC utilized a typical

Table I Demographic characteristics

\begin{tabular}{llll}
\hline & Group A & Group B & Total \\
\hline $\begin{array}{llll}\text { Gender } \\
\text { Female }\end{array}$ & $40 I(58.28 \%)$ & $123(62 \%)$ & $688(100 \%)$ \\
Male & $287(41.71 \%)$ & $77(39 \%)$ & $200(100 \%)$ \\
Age & & & \\
$n$ & 688 & 200 & 888 \\
Mean & 41.8 & 47.7 & 43.13 \\
SD & 11.35 & 19.14 & 13.71 \\
\hline
\end{tabular}

Abbreviation: SD, standard deviation. 
Table 2 Number needed to treat analysis

\begin{tabular}{llll}
\hline & Present & Absent & Total \\
\hline Given (Group A) & 196 & 496 & 688 \\
Not given (Group B) & 135 & 65 & 200 \\
Total & $33 \mathrm{I}$ & 557 & 888 \\
Risk of outcome in treated group & 0.2848 & \\
Risk of outcome in control group & 0.675 & \\
Absolute risk reduction & $0.390 \mathrm{I}$ & \\
Relative risk & 0.4220 & \\
Relative risk reduction & 0.5779 & \\
Number needed to treat & & 2.5633 & \\
\hline
\end{tabular}

range of frequencies of $2.4,5.8$, or $10.5 \mathrm{gHz}$, as selected by the operator for each specific protocol. In the brain stimulation protocols used in this study, a frequency of $10.5 \mathrm{gHz}$ was used with a specific absorption rate of $7 \mathrm{~mW} / \mathrm{kg}$. A sequence of seven radiofrequency pulses of 500 milliseconds, termed the neuropsychophysical optimization protocol, was applied by touching the metallic tip of the REAC probe onto seven specific reflex auricular points. This protocol consists of 18 sessions, usually administered on alternate days. The goal of neuropsychophysical optimization is gradual amelioration of symptoms of both physical and mental dysfunction. To achieve this, the amount of treatment given is in relation to symptom severity. The neuropsychophysical optimization treatment is painless, simple, rapid, and noninvasive. The REAC model used in this study was the Convogliatore di Radianza Modulante (ASMED, Italy).

\section{Statistical analysis}

Statistical analysis was performed using number needed to treat analysis (Table 2). To compare total points before and after treatment or placebo, the Wilcoxon signed-rank test was used, while the McNemar test was used to test for the presence of symptoms of pain and physical problems. $P<0.05$ was considered to be statistically significant.

\section{Results}

Prior to REAC treatment, 403 (58.58\%) patients in Group A were positive for stress-related pain and physical problems.
In Group B, pain and physical problems were detected in 159 (79\%) patients. Following REAC treatment, only 196 of 403 patients (48.63\%) in Group A still presented symptoms of stress-related pain and physical problems (McNemar Chisquare test $=170.426$, asymptotic significance $=0.000)$. In Group B, pain and physical problems were observed in 135 of 159 subjects $(84.90 \%)$. Total point scores decreased significantly in Group A subjects following treatment, from 122.3 to 96.01 (Wilcoxon signed-rank test $Z=-22.735$, asymptotic significance [two-tailed] $=0.000$, Table 3 ). The decrease in total point scores from 122.96 to 122.11 in Group B was not statistically significant (Wilcoxon signed-rank test $\mathrm{Z}=-0.914$, asymptotic significance [two-tailed] $=0.361$, Table 3). There was no significant effect of age or gender upon therapy-induced changes in total points.

\section{Discussion}

The REAC reshapes electrical changes due to ion flows, ${ }^{35}$ which likely balances the distributions of two main neurotransmitters, ie, excitatory glutamate ${ }^{36}$ and inhibitory gamma aminobutyric acid. ${ }^{37}$ The neuropsychophysical optimization protocol induces a new positive regulation of bioelectric activity in the central nervous system, leading to normalization of brain function that can be tailored to the individual, ${ }^{22-34,38}$ according to symptoms of pain and physical problems. The current study was designed based upon the results of a previous study that demonstrated the beneficial effects of REAC in stress-related disorders, ${ }^{24}$ and was targeted to assess specific stress-related clusters on the PSM test. ${ }^{25}$ The perception of acute and chronic pain may be influenced by stress and the psychological state of sufferers, often without awareness. ${ }^{39-50}$ This unrelieved pain may cause the sufferer to use or abuse narcotic drugs, and physicians may prescribe painkillers or tranquilizers to reduce the tension associated with pain. ${ }^{51}$ Therefore, a targeted therapy that improves the neuropsychophysical response to environmental stressors could represent a viable alternative to pharmaceutical treatments. REAC is a noninvasive, drug-free alternative

Table 3 Psychological Stress Measure test results

\begin{tabular}{llll}
\hline Subjects & Total points PSM test & PPP subjects (\%) & Wilcoxon test \\
\hline Group A $(n=688), T_{0}$ & $122.53 \pm 6.747$ & 403 & Asymp sig (two-tailed) 0.000 \\
Group A, $T_{1}$ & $96.01 \pm 8.520$ & $196(48 \%)$ & $Z=-22.735 P<0.005$ \\
Group B $(n=200), T_{0}$ & $122.96 \pm 7.041$ & 159 & Asymp sig (two-tailed) 0.361 \\
Group B, $T_{1}$ & $122.11 \pm 7.450$ & $135(85 \%)$ & $Z=-0.914 P>0.005$ \\
\hline
\end{tabular}

Notes: PSM test results: Total points and Wilcoxon test results obtained in Group A and in Group B, before $\left(T_{0}\right)$ and after $\left(T_{1}\right)$ NPPO REAC treatment/placebo. Values of total points are expressed by mean \pm standard deviation. $* P<0.05$.

Abbreviations: NPPO, neuropsychophysical optimization; REAC, radioelectric asymmetric conveyer; PSM, Psychological Stress Measure; PPP, pain and physical problems; asymp sig, asymptotic significance. 
for the treatment of pain, particularly in situations where emotional state and stress levels of the individual clearly affect overall symptomatology.

\section{Conclusion}

This research highlights the efficacy of REAC neuropsychophysical optimization on the pain and physical symptoms cluster measurable using the PSM test. The pain and physical symptom cluster represents a set of disorders that can severely impact quality of life in a large proportion of the population. For this reason, it is necessary to find new therapeutic strategies able to treat the various components that underlie these symptoms. Of course, because symptoms of pain and physical problems are often chronic, it is desirable that efficacious treatments also be safe and cost-effective. Further studies are needed to verify the stability of symptoms over time when using more than one cycle of REAC neuropsychophysical optimization, although there may be difficulties in obtaining and following a selected group of patients, especially after treatment. A longer period of observation and administration of additional REAC therapy cycles is necessary to assess the stability of their therapeutic effects over time.

\section{Acknowledgments}

The authors thank Barbara Rosettani for her help with the statistical analysis and Stefania Bini for her helpful discussions.

\section{Disclosure}

SR and VF are the inventors of the radioelectric asymmetric conveyer.

\section{References}

1. Lempa M, Grotemeyer KH, Hauser W, Moormann O, Wesselmann U, Derra C. Dysfunctional syndromes: their diagnostics and treatment. Schmerz. 2004;18:141-144. German.

2. Dirkzwager AJ, Verhaak PF. Patients with persistent medically unexplained symptoms in general practice: characteristics and quality of care. BMC Fam Pract. 2007;8:33.

3. Reid S, Wessely S, Crayford T, Hotopf M. Medically unexplained symptoms in frequent attenders of secondary health care: retrospective cohort study. BMJ. 2001;322:767.

4. van Dieren Q, Vingerhoets AJ. Medically unexplained somatic symptoms are not unexplainable, misunderstood or vague physical complaints. Tijdschr Psychiatr. 2007;49:823-834. Dutch.

5. Kisely S, Simon G. An international study comparing the effect of medically explained and unexplained somatic symptoms on psychosocial outcome. J Psychosom Res. 2006;60:125-130.

6. Escobar JI, Hoyos-Nervi C, Gara M. Medically unexplained physical symptoms in medical practice: a psychiatric perspective. Environ Health Perspect. 2002;110 Suppl 4:631-636.

7. Salmon P, Humphris GM, Ring A, Davies JC, Dowrick CF. Primary care consultations about medically unexplained symptoms: patient presentations and doctor responses that influence the probability of somatic intervention. Psychosom Med. 2007;69:571-577.
8. Salmon P, Wissow L, Carroll J, et al. Doctors' attachment style and their inclination to propose somatic interventions for medically unexplained symptoms. Gen Hosp Psychiatry. 2008;30:104-111.

9. Marcus H, Aldam P, Lennox G, Laing R. Medically unexplained neurological symptoms. JRSM Short Rep. 2010;1:25.

10. Fornaro M, Maremmani I, Canonico PL, et al. Prevalence and diagnostic distribution of medically unexplained painful somatic symptoms across 571 major depressed outpatients. Neuropsychiatr Dis Treat. 2011;7:217-221.

11. Hilderink PH, Benraad CE, van Driel D, et al. Medically unexplained physical symptoms in elderly people: a pilot study of psychiatric geriatric characteristics. Am J Geriatr Psychiatry. 2009;17:1085-1088.

12. Hilderink PH, Benraad CE, van Driel TJ, Olde Rikkert MG. Medically unexplained symptoms in older adults: a combination of physical, psychiatric and psychological factors. Ned Tijdschr Geneeskd. 2008;152:1305-1308. Dutch.

13. Bussing A, Ostermann T, Neugebauer EA, Heusser P. Adaptive coping strategies in patients with chronic pain conditions and their interpretation of disease. BMC Public Health. 2010;10:507.

14. McEwen BS. The brain is the central organ of stress and adaptation. Neuroimage. 2009;47:911-913.

15. McEwen BS, Gianaros PJ. Central role of the brain in stress and adaptation: links to socioeconomic status, health, and disease. Ann NY Acad Sci. 2010;1186:190-222.

16. American Psychiatric Association. Diagnostic and Statistical Manual of Mental Disorders. Fourth Edition, Text Revision. Washington, DC: American Psychiatric Association; 2000.

17. Lemyre L, Tessier R. Measuring psychological stress. Concept, model, and measurement instrument in primary care research. Can Fam Physician. 2003;49:1159-1160.

18. Lemyre LTR. Measurement of psychological stress: To feel stressed. Rev Canad Sci Comport. 1988;20:302-321. French.

19. Trovato GM, Catalano D, Martines GF, et al. Psychological stress measure in type 2 diabetes. Eur Rev Med Pharmacol Sci. 2006;10:69-74.

20. Rinaldi S, Fontani V, inventor; Rinaldi S, Fontani V, assignee. Radioelectric Asymmetric Conveyer for therapeutic use. EP1301241. October 11, 2006, 2000.

21. Rinaldi S, Fontani V, inventor; Rinaldi S, Fontani V, assignee. Radioelectric Asymmetric Conveyer for therapeutic use. US patent 7,333,859. February 19 2008, 2001.

22. Collodel G, Moretti E, Fontani V, et al. Effect of emotional stress on sperm quality. Indian J Med Res. 2008;128:254-261.

23. Rinaldi S, Fontani V, Aravagli L, Margotti ML. Psychological and symptomatic stress-related disorders with radio-electric treatment: psychometric evaluation. Stress and Health. 2010;26:350-358.

24. Rinaldi S, Fontani V, Aravagli L, Mannu P. Psychometric evaluation of a radio electric auricular treatment for stress related disorders: a doubleblinded, placebo-controlled controlled pilot study. Health Qual Life Outcomes. 2010;8:31.

25. Rinaldi S, Fontani V, Moretti E, et al. A new approach on stressrelated depression and anxiety: neuro-psycho-physical-optimization with radio electric asymmetric-conveyer. Indian J Med Res. 2010;132:189-194.

26. Mannu P, Rinaldi S, Fontani V, Castagna A, Margotti ML. Radio electric treatment vs es-citalopram in the treatment of panic disorders associated with major depression: an open-label, naturalistic study. Acupunct Electrother Res. 2009;34:135-149.

27. Olivieri EB, Vecchiato C, Ignaccolo N, et al. Radioelectric brain stimulation in the treatment of generalized anxiety disorder with comorbid major depression in a psychiatric hospital: a pilot study. Neuropsychiatr Dis Treat. 2011;7:449-455.

28. Mannu P, Rinaldi S, Fontani V, Castagna A. Long-term treatment of bipolar disorder with a radioelectric asymmetric conveyor. Neuropsychiatr Dis Treat. 2011;7:373-379.

29. Mannu P, Rinaldi S, Fontani V, Castagna A. Radio electric asymmetric brain stimulation in the treatment of behavioral and psychiatric symptoms in Alzheimer disease. Clin Interv Aging. 2011;6:207-211. 
30. Castagna A, Rinaldi S, Fontani V, Mannu P. Radioelectric asymmetric brain stimulation and lingual apex repositioning in patients with atypical deglutition. J Multidiscip Healthc. 2011;4:209-213.

31. Castagna A, Fontani, V, Rinaldi S, Mannu P. Radio electric tissue optimization in the treatment of surgical wounds. Clin Cosmet Investig Dermatol. 2011;4:133-137.

32. Fontani V, Castagna A, Mannu P, Rinaldi S. Radioelectric asymmetric stimulation of tissues as treatment for post-traumatic injury symptoms. Int J Gen Med. 2011;4:627-634.

33. Castagna A, Rinaldi S, Fontani V, Mannu P, Margotti ML. Comparison of two treatments for coxarthrosis: local hyperthermia versus radio electric asymmetrical brain stimulation. Clin Interv Aging. 2011;6(1): 201-206.

34. Castagna A, Rinaldi S, Fontani V, Aravagli L, Mannu P, Margotti ML. Does osteoarthritis of the knee also have a psychogenic component? Psycho-emotional treatment with a radio-electric device vs intraarticular injection of sodium hyaluronate: an open-label, naturalistic study. Acupunct Electrother Res. 2010;35:1-16.

35. Clark VP, Coffman BA, Trumbo MC, Gasparovic C. Transcranial direct current stimulation (tDCS) produces localized and specific alterations in neurochemistry: A (1)H magnetic resonance spectroscopy study. Neurosci Lett. 2011;500:67-71.

36. Biedermann $\mathrm{S}$, Weber-Fahr W, Zheng L, et al. Increase of hippocampal glutamate after electroconvulsive treatment: A quantitative proton MR spectroscopy study at $9.4 \mathrm{~T}$ in an animal model of depression. World $J$ Biol Psychiatry. July 18, 2011. [Epub ahead of print].

37. Pamenter ME, Hogg DW, Ormond J, Shin DS, Woodin MA, Buck LT. Endogenous GABA(A) and GABA(B) receptor-mediated electrical suppression is critical to neuronal anoxia tolerance. Proc Natl Acad Sci U S A. 2011;108:11274-11279.

38. Rinaldi S, Fontani V, Aravagli L, et al. Stress-related psycho-physiological disorders: randomized single blind placebo controlled naturalistic study of psychometric evaluation using a radio electric asymmetric treatment. Health Qual Life Outcomes. 2011;9:54.

39. Anderson RU, Orenberg EK, Morey A, Chavez N, Chan CA. Stress induced hypothalamus-pituitary-adrenal axis responses and disturbances in psychological profiles in men with chronic prostatitis/chronic pelvic pain syndrome. J Urol. 2009;182:2319-2324.

40. Burns JW. The role of attentional strategies in moderating links between acute pain induction and subsequent psychological stress: evidence for symptom-specific reactivity among patients with chronic pain versus healthy nonpatients. Emotion. 2006;6(2):180-192.
41. Dorn LD, Campo JC, Thato S, et al. Psychological comorbidity and stress reactivity in children and adolescents with recurrent abdominal pain and anxiety disorders. J Am Acad Child Adolesc Psychiatry. 2003;42(1):66-75

42. Greco CM, Rudy TE, Manzi S. Effects of a stress-reduction program on psychological function, pain, and physical function of systemic lupus erythematosus patients: a randomized controlled trial. Arthritis Rheum. 2004;51(4):625-634.

43. McLean SA, Clauw DJ, Abelson JL, Liberzon I. The development of persistent pain and psychological morbidity after motor vehicle collision: integrating the potential role of stress response systems into a biopsychosocial model. Psychosom Med. 2005;67:783-790.

44. Olbrich D, Ruch A. Diagnosis in back pain involves not only the body. Investigate psychological stress to determine origin. MMW Fortschr Med. 2001;143:22-25. German.

45. Solowiej K, Mason V, Upton D. Psychological stress and pain in wound care, part 2: a review of pain and stress assessment tools. JWound Care. 2010;19:110-115.

46. Starkweather AR, Witek-Janusek L, Nockels RP, Peterson J, Mathews HL. Immune function, pain, and psychological stress in patients undergoing spinal surgery. Spine (Phila Pa 1976). 2006;31(18): E641-E647.

47. Truchon M, Cote D, Fillion L, Arsenault B, Dionne C. Low-back-pain related disability: an integration of psychological risk factors into the stress process model. Pain. 2008;137:564-573.

48. van Selms MK, Lobbezoo F, Visscher CM, Naeije M. Myofascial temporomandibular disorder pain, parafunctions and psychological stress. J Oral Rehabil. 2008;35:45-52.

49. van Selms MK, Lobbezoo F, Wicks DJ, Hamburger HL, Naeije M. Craniomandibular pain, oral parafunctions, and psychological stress in a longitudinal case study. J Oral Rehabil. 2004;31:738-745.

50. Yoshihara T, Shigeta K, Hasegawa H, Ishitani N, Masumoto Y, Yamasaki Y. Neuroendocrine responses to psychological stress in patients with myofascial pain. J Orofac Pain. 2005;19:202-208.

51. Pergolizzi J, Alon E, Baron R, et al. Tapentadol in the management of chronic low back pain: a novel approach to a complex condition? J Pain Res. 2011;4:203-210. 


\section{Supplement I}

This trial was registered with the Australian New Zealand Clinical Trials Registry (ANZCTR) with the number ACTRN12607000456459. Registration is available at the following link: http://www.anzctr.org.au/trial_view. aspx?ID=82252.

\section{Publish your work in this journal}

The International Journal of General Medicine is an international, peer-reviewed open-access journal that focuses on general and internal medicine, pathogenesis, epidemiology, diagnosis, monitoring and treatment protocols. The journal is characterized by the rapid reporting of reviews, original research and clinical studies across all disease areas.
A key focus is the elucidation of disease processes and management protocols resulting in improved outcomes for the patient.The manuscript management system is completely online and includes a very quick and fair peer-review system. Visit http://www.dovepress.com/ testimonials.php to read real quotes from published authors. 